\title{
Inflammatory response in Parkinson's disease (Review)
}

\author{
JUNQIANG YAN*, QIZHI FU*, LINIU CHENG, MINGMING ZHAI, \\ WENJUAN WU, LINA HUANG and GANQIN DU

\begin{abstract}
Department of Neurology, The First Affiliated Hospital of Henan University of Science and Technology,
\end{abstract} \\ Luoyang, Henan 471003, P.R. China
}

Received December 12, 2013; Accepted July 1, 2014

DOI: $10.3892 / \mathrm{mmr} .2014 .2563$

\begin{abstract}
Parkinson's disease (PD) is one of the most common age-related neurodegenerative diseases, which results from a number of environmental and inherited factors. PD is characterized by the slow progressive degeneration of dopaminergic (DA) neurons in the substantia nigra. The nigrostriatal DA neurons are particularly vulnerable to inflammatory attack. Neuroinflammation is an important contributor to the pathogenesis of age-related neurodegenerative disorders, such as PD, and as such anti-inflammatory agents are becoming a novel therapeutic focus. This review will discuss the current knowledge regarding inflammation and review the roles of intracellular inflammatory signaling pathways, which are specific inflammatory mediators in PD. Finally, possible therapeutic strategies are proposed, which may downregulate inflammatory processes and inhibit the progression of PD.
\end{abstract}

\section{Contents}

1. Introduction

2. Cellular response to Parkinson's disease

3. Inflammatory and anti-inflammatory mediators

4. Transcriptional regulation of inflammatory processes

5. Conclusion

\section{Introduction}

Parkinson's disease (PD) is a neurodegenerative, multifactorial movement disorder affecting $\sim 3 \%$ of the population aged $>65$ years (1). As a neurodegenerative disorder, PD is the

Correspondence to: Professor Junqiang Yan, Department of Neurology, The First Affiliated Hospital of Henan University of Science and Technology, 24 Jinghua Road, Luoyang, Henan 471003, P.R. China

E-mail: yanjq20062007@126.com

*Contributed equally

Key words: inflammation, Parkinson's disease, NR4A, DJ-1, toll-like receptors, cytokines second most common cause of age-linked neurodegeneration after Alzheimer's disease (AD). Furthermore, PD is one of the most common causes of disability and mortality globally; hence it has a significant clinical and socioeconomic impact. The primary pathological characteristic of PD is the chronic and progressive loss of dopaminergic (DA) neurons in the substantia nigra pars compacta (SNPC), which leads to irreversible striatal dopamine loss. The symptoms of PD are only observed once the dopamine levels in the striatum are reduced by $>80 \%$.

While the pathogenesis of PD is not fully understood, increasing evidence from previous studies has revealed that inflammatory responses are responsible for the progression of PD (2-5). These responses include accumulation of inflammatory mediators, such as inflammatory cytokines and proteases in the substantia nigra and the striatum, as well as activation of the microglia. While it has not yet been demonstrated at the clinical level that decreasing inflammatory cascades ameliorates DA neuronal injury, numerous studies have demonstrated this effect in models of PD (6-9).

Recently, marked progress has been made in identifying the roles of inflammatory signaling molecules, cells and proteins in the initiation and development of PD. This review focuses on summarizing the novel data regarding the inflammatory response in PD.

\section{Cellular response to Parkinson's disease}

Inflammation is the accumulation of inflammatory cells and mediators in the brain. Studies of the chronic inflammatory processes involved in PD have found that microglia and astrocytes have important roles in the occurrence of inflammation.

Microglia/macrophages. Microglia are resident immunocompetent and phagocytic cells within the brain and, as such, are significant in the pathogenesis and progression of PD. Once microglia are activated, they undergo a morphological transformation into phagocytes, which makes them indistinguishable from macrophages. In addition, the activation of microglia leads to progressive inflammation and neuronal cell death, causing further reactive microglia activation, which may lead to a cycle of inflammation and neuronal injury that fuels the progressive DA neurodegeneration in PD $(10,11)$. Excessive levels of microglial activation have been identified in the SNPC of patients with PD (12). Since the substantia nigra has the highest 
density of microglia (13) and is more susceptible to inflammatory attack, microglial activation may damage the DA neurons within the substantia nigra. Furthermore, levels of intracellular glutathione in DA neurons are lower than those in other areas of the substantia nigra, which reduces the antioxidant ability of the DA neurons and increases their sensitivity to microglial activation and oxidative stress (14). A previous study revealed that the number of activated microglia increased markedly in the SNPC of 6-hydroxydopamine (OHDA)-lesioned PD rat models at 10 and 28 days post-lesion (15). All these results indicate that microglial cells are involved in the pathogenesis of PD in numerous different ways.

Though the mechanism by which microglial cells are activated in PD remains unknown, accumulating evidence indicates that the activation of microglia is associated with a series of neurochemical mediators. Microglia of the substantia nigra can be activated by interferon (IFN) $-\gamma$, inducible nitric oxide synthase (iNOS), interleukin (IL)-1 $\beta$ and tumor necrosis factor (TNF)- $\alpha$. In the progression of PD, microglia activation is associated with cyclooxygenase (COX)-2, complement receptor 3 and increased levels of ferritin $(16,17)$. Nitration or oxidative stress modifies certain surface proteins on DA neurons enabling the reconstruction of those surface proteins and their recognition as foreign pathogenic antigens by microglia, which are subsequently activated $(18,19)$.

Notably, Orr et al (20) found that activated microglia, within the first $24 \mathrm{~h}$ of stimulus disturbance, increaseses immunoglobulin reactivity, and upregulates the expression levels of cell adhesion molecules. In the substantia nigra of PD patients and animal models, the expression levels of iNOS, an important oxidative stress mediator, are also increased (18). iNOS is colocalized with the activation and multiplication of microglia cells, which implies that nitric oxide (NO) induces protein upregulation and increases levels of nitrotyrosine production $(21,22)$. The induction of proinflammatory and immune system cytokines, such as IFN- $\gamma$, TNF- $\alpha$ and IL-1 promotes iNOS production (23) and aggravates NO-induced DA neuronal injury. Subsequently, proinflammatory cytokines accelerate the activation of microglia and their infiltration across the blood-brain barrier, which leads to the activation of further, resident microglia and perivascular macrophages (24), resulting in a chronic inflammatory response. When this inflammatory response persists, it may cause the progression of PD.

In conclusion, these results indicate that activated microglia are beneficial in the early stages of PD, however, with the development of the disease the activated microglia begin to exert a neurotoxic effect.

Astrocytes. Astrocytes are important mediators in the homeostasis of the extracellular environment of neurons (25). Increasing evidence has revealed that the role of astrocytes within the brain may be either protective or detrimental. Whilst certain neurodegenerative diseases may be caused by over-reactive astrocytes (26), they may also inhibit oxidative stress, prevent mitochondrial damage and produce neurotrophic factors (27). Astrocytes have a protective effect on DA neurons by increasing the expression levels of glial fibrillary acidic protein (GFAP) and glutathione peroxidase (28). Activating the nuclear factor of $\kappa$ light polypeptide gene enhancer in
$\mathrm{B}$ cells $(\mathrm{NF}-\kappa \mathrm{B})$ of reactive astrocytes in the substantia nigra can lead to DA neuronal loss in PD model mice treated with 1-methyl-4-phenyl-1,2,3,6-tetrahydropyridine (MPTP) (29). Activation of astrocytes counteracts 6-OHDA-mediated toxicity in nigral DA cell bodies (30) and releases factors that are toxic to neurons in the inflammation-mediated pathogenesis of neurological diseases, such as AD and PD (31). In mesencephalic DA neuronal cultures, astrocytes promote mesencephalic neuronal survival and reduce the presence of apoptotic nuclear profiles (32). In PD, the activation of brain astrocytes increases the expression levels of GFAP, an astrocytic marker, which results in specific structural and functional changes (33). In a 6-OHDA-induced PD rat model, a marked increase in the immunoreactivity of GFAP was displayed in the SNPC and the striatum (34).

While the mechanism of action of astrocytes in brain inflammation has not been fully elucidated in PD models, certain studies have demonstrated that astrocytes lead to inflammatory reaction by developing type 2 helper $\mathrm{T}$ cell (Th2) immune responses, suppressing IL-12 expression and expressing major histocompatibility complex (MHC) and costimulatory molecules (35). In certain diseases, such as stroke, multiple sclerosis and PD, astrocytes express aberrant inflammatory factors, including chemokines, cytokines and iNOS. The severity of DA neuronal death is hypothesized to be parallel to $\alpha$-synuclein-positive astrocytes in PD (36).

In summary, these studies indicate that activated astrocytes have the capacity to produce neuroprotective effects in PD. Therefore, a pharmacological manipulation of these mechanisms may be a possible therapeutic strategy to investigate.

Oligodendrocytes. Oligodendrocytes are a type of neuroglia whose predominant function is the insulation of axons in the central nervous system (CNS). They are the cells that are responsible for the synthesis and assembly of myelin, which surrounds the axons in the CNS. Previous studies have demonstrated that oligodendrocytes are key in certain neurodegenerative diseases $(37,38)$, however, there are few studies regarding oligodendrocytes in $\mathrm{PD}$.

Takagi et al (39) found that the area of expression of 2',3'-cyclic nucleotide 3' phosphodiesterase (CNP)-positive profiles and the expression levels of CNPase protein were markedly reduced in the striatum three and seven days after MPTP treatment compared with those in the control groups, demonstrating that oligodendrocytes may be involved in an MPTP-mediated neurotoxic role in PD. Dopamine D2 and D3 receptors are expressed in rat differentiated cortical oligodendrocytes, and dopamine D2 and D3 receptor-like immunoreactivity has additionally been found in cultured oligodendrocytes (40). Oligodendrocytes and their precursors are highly susceptible to excitotoxic damage, oxidative stress and the effects of cytokines (41); furthermore, the activation of dopamine D2 and D3 receptors may protect oligodendrocytes against glutamate oxidative stress-induced cell death. Notably, increased expression levels of D2 and D3 receptor proteins were observed after oligodendrocytes were exposed to oxygen/glucose deprivation for $2 \mathrm{~h}$. Immunization of mice with the myelin oligodendrocyte glycoprotein prior to treatment with MPTP significantly reduces microglial reactivity and the expression levels of proinflammatory cytokines, whilst 
elevating the expression levels of glial cell line derived neurotrophic factor (GDNF) mRNA compared with those observed in mice treated with MPTP only (42).

Whilst there has been limited research into the association between oligodendrocytes and PD, it is hypothesized that oligodendrocyte may have a neuroprotective role, which may contribute to the elucidation of the progressive mechanisms of PD. However, further studies are required to provide evidence of this in animal models and in patients with PD.

\section{Inflammatory and anti-inflammatory mediators}

Cytokines. Cytokines are upregulated in the brain as a result of chronic inflammation, such as that observed in PD. In the cells of the immune system the resident brain cells, including microglia and neurons, express and produce cytokines. In PD the most investigated cytokines are IL-1, IL-6, IL-10, IFN- $\gamma$, transforming growth factor (TGF) $\beta$ and TNF- $\alpha$.

$T N F-\alpha$. TNF- $\alpha$ is an important proinflammatory cytokine. Expression levels of TNF- $\alpha$ have been observed to be higher in the striatum and substantia nigra of 6-OHDA-induced PD model rats compared with those in the control rats. In the nigrostriatal signaling pathway, which links the substantia nigra and the striatum, TNF- $\alpha$ expression can be upregulated, which is accompanied by microglial activation. Additionally, increased levels of TNF- $\alpha$ expression have been observed in the SNPC of patients with PD (11). TNF- $\alpha$ knockout (KO) in MPTP-mediated PD model mice demonstrated reduced blood-brain barrier leakage and MPTP-induced inflammation when compared with that in the wild-type controls (43). Reduced levels of methylation of the TNF- $\alpha$ promoter in SNPC cells increases the susceptibility of DA neurons to TNF- $\alpha$-mediated inflammatory reactions (44).

TNF- $\alpha$ acts via at least two TNF- $\alpha$ receptors in the development of PD: TNF receptor 1 (TNFR1) and TNF receptor 2 (TNFR2). Following MPTP injection in TNFR1 and TNFR2 double KO mice, the level of dopamine expression in the striatum was observed to be reduced, however, the dopamine turnover was increased (45). These results concur with those of Sriram et al $(46,47)$. The levels of TNF- $\alpha$ and the immunoreactivity of TNFRs were markedly enhanced in the substantia nigra, striatum and cerebrospinal fluid (CSF) of patients with PD. Furthermore, it has been identified that TNFR double KO mice experience inhibited microglia activation and protection against MPTP-induced DA neurotoxicity. Injection of TNF- $\alpha$ was demonstrated to result in dopamine neuron loss in the SNPC of rodents. Blocking the soluble form of the TNF- $\alpha$ receptor reduces the level of DA neuron death in 6-OHDA-lesioned rats (48). A study into the serum levels of TNF- $\alpha$ and the soluble forms of their receptors (sTNFRs) in patients with PD (49) revealed that patients with PD had higher levels of sTNFR1 compared with those observed in the healthy controls, which was associated with a later disease onset (50).

However, another study revealed that TNF- $\alpha \mathrm{KO}$ only suppresses MPTP-induced microglial activation and does not reduce the loss of dopamine neurons caused by MPTP treatment. This indicates that in the progression of PD, the loss of dopamine neurons is not directly caused by TNF- $\alpha$, rather it acts as a mediator, interacting with the TNFR1 and TNFR2 subtypes (43).

$I L-1$. IL-1 was one of the first cytokines to be described. It is an upstream signaling molecule for a number of inflammatory factors and neurotoxic mediators. Microglial activation leads to a number of phenotypes, one of which is a proinflammatory phenotype. The secretion of IL-1 $\beta$ has a similar proinflammatory effect, which can be reversed by specific IL-1 inhibition. The IL-1 receptor antagonist significantly attenuates the augmented loss of DA neurons caused by lipopolysaccharide (LPS) or 6-OHDA-induced sensitization to DA degeneration.

IL has two isoforms, IL-1 $\alpha$ and IL-1 $\beta$. The endogenous inhibitor of IL-1 $\alpha$, IL-1 $\beta$ and IL has been widely investigated in PD. In the ventral midbrain and substantia nigra of MPTP-induced animal models the expression levels of IL-1 $\beta$ were elevated, as was the activation of microglia (51). Blocking microglial activation as well as altering the shape of the tertiary structure of mature IL-1 $\beta$ causing inhibition of its activity can reduce MPTP-induced DA neurotoxicity. The action of a single proinflammatory cytokine, for example IL-1 $\beta$, could lead to dopamine neuron loss independent of other inflammatory events. In the substantia nigra of adult rats, a recombinant adenovirus expressing IL-1 $\beta$ was used in a previous study to induce the chronic expression of IL- $1 \beta$, leading to DA cell loss and motor disorder (52). In the CSF and nigrostriatal regions of clinical patients with PD, the IL-1 $\beta$ expression levels were found to be markedly increased (53-55). Notably, compared with the expression levels of IL- $1 \beta$, the IL- $1 \alpha-889$ polymorphism did not demonstrate a significant increase between the patients with PD and the control subjects. The reasons and mechanisms behind the different effects of IL- $1 \alpha$ and IL-1 $\beta$ on PD patients have yet to be elucidated.

$I F N-\gamma$. IFN- $\gamma$ is secreted by $\mathrm{T}$ lymphocytes and natural killer cells, and is a cytokine for innate and adaptive immunity against viral and intracellular bacterial infections. In MPTP-induced rat models, a significant increase of IFN- $\gamma$ has been observed in the striatum within one week of administration of MPTP. In the study of in vivo and in vitro models of $\mathrm{PD}$, it has been demonstrated that PD patients exhibit elevated IFN-plasma levels compared with the levels observed in non-PD patients. Additionally, MPTP-induced DA cell loss was significantly attenuated in IFN- $\gamma$ deficient mice, which was accompanied by a reduction in striatal tyrosine hydroxylase $(\mathrm{TH})$ and dopamine transporter fiber density. Furthermore, the levels of DA and 3,4-dihydroxyphenylacetic acid (DOPAC) were significantly reduced in MPTP-treated wild-type mice. However, the loss of DA neurons was significantly reduced in the MPTP-treated IFN- $\gamma$ KO mice (56). IFN- $\gamma$ deficiency attenuates the reduction of striatal DA levels and behavioral effects following paraquat exposure (57). These results demonstrate that IFN- $\gamma$ is involved in the death of DA neurons in the development of PD, which is associated with the regulation of microglial activity. Numerous clinical studies have demonstrated that the expression level of IFN- $\gamma$ was significantly elevated in the blood plasma of patients with PD (45), and that levels were higher in the nigrostriatal DA regions, including the substantia nigra, caudate nucleus and putamen, of PD patients than those in the 
control subjects (58). The clinical findings also revealed that the elevation of IFN- $\gamma$ expression levels in the blood serum and the brain led to the degeneration of the nigrostriatal DA neurons in PD.

Other inflammation-related cytokines. IL-6 is a common proinflammatory cytokine, however, it is not clear whether IL-6 also has a significant role in PD. A nested case-control study found that males with high plasma concentrations of IL-6 have an increased risk of developing PD (59). Scalzo et al (50) found that serum levels of IL-6 were markedly increased in patients with $\mathrm{PD}$, and that PD patients who exhibited higher serum levels of IL-6 demonstrated a reduced gait speed, more problems in performing tasks that required postural changes and reduced base support (50), indicating that IL-6 may be associated with motion function in patients with PD.

IL-10, an anti-inflammatory cytokine, inhibits IL-1 and TNF- $\alpha$, and suppresses cytokine receptor expression and receptor activation. Previous studies have revealed that the expression level of IL-10 is constant in the CSF and serum of PD patients (60). However, a recent study found that patients with PD exhibited significantly increased IL-10 expression levels compared with those observed in the control group (61). Contrary to IL-6, IL-10 appears to exert beneficial effects in the progression of PD. In an LPS-induced mouse model of PD, intracerebral IL-10 was shown to reduce the number of activated microglia and inhibit LPS-mediated production of TNF- $\alpha$ and NO, subsequently leading to neuroprotection against LPS-induced DA neuronal death (62). In the presence of microglia, IL-10 has been reported to protect reconstituted neuron and glial cell cultures from LPS-induced neurotoxicity, however, these protective effects vanish in mice that lack nicotinamide adenine dinucleotide phosphate (NADPH) oxidase (63). These studies indicate that through the inhibition of microglial activation, IL-10 may exhibit neuroprotective effects that reduce the damage to DA neurons.

TGF- $\beta 1$ has been detected in microglia and astrocytes, and has been found to be expressed at low levels in the neurons of healthy subjects (64). In addition, upregulation of TGF- $\beta 1$ has been observed in the brains of animals with neurodegenerative disease. In the absence of MPTP treatment, infusion of TGF- $\beta 1$ into the ventral mesencephalon of mice causes a detrimental effect to DA neurons (65). TGF- $\beta 1$ exerts a significant neuroprotective effect in LPS- and MPTP-mediated models of PD through its anti-inflammatory properties (66).

Chemokines and chemokine receptors. Chemokines are a family of potent, soluble, chemoattractant cytokines that are involved in leukocyte migration, angiogenesis, cell activation, cell proliferation and cell differentiation. According to the positions of the cysteine residues (C) within them, chemokines have been classified as follows: CXC, CC, C and CX3C. Each class has a different role, which it undertakes via specific and shared receptors that belong to the superfamily of $\mathrm{G}$ protein-coupled receptors. Monocyte chemotactic protein 1 (MCP-1) is a ligand of the CC class of chemokines. In MPTP-lesioned mice, the level of expression of the MCP-1 mRNA was found to be significantly increased in the striatal region, resulting in damage to TNF receptor-deficient DA neurons (46). Additionally, the level of expression of IL-8
mRNA was observed to be increased in the blood serum of PD patients (67). Clinical studies have found that the age of onset of PD in patients may be associated with the 2518G/A genotype in the CCL2/MCP-1 promoter region (68). Significantly increased expression levels of the CXCR4 chemokine have been observed in the DA neurons of the substantia nigra in humans. Notably, greater expression levels of CXCR4 and CXCL12 were observed in the post-mortem brains of PD patients when compared with those in the control group, despite the loss of DA neurons. In addition, the same study reported that MPTP produced a time-dependent upregulation of CXCR4 prior to the loss of DA neurons in the substantia nigra of MPTP-treated mice (69). Edman et al (70) demonstrated that $\alpha$-chemokines, including CXCL1, CXCL6 and CXCL8, increase the number of DA neurons in the ventral midbrain precursor of a developing rat. These findings indicate that chemokines and chemokine receptors may be involved in the etiology of PD.

Cyclooxygenase (COX) signaling pathway. COX converts arachidonic acid, which is released from brain phospholipids during the progression of $\mathrm{PD}$, into prostaglandin (PG) H2 . COX has three isoforms: COX-1, COX-2 and COX-3. Within the three subtypes of COX, it is COX-2 rather than COX-1 or COX-3 that has the most important role in the development of PD. COX-2 expression in the substantia nigra of mice with MPTP-induced PD was found to be significantly increased in the DA neurons and microglia of the striatum and ventral midbrain $(6,71,72)$. In an MPTP-induced mouse model of PD, blocking COX-2 has been demonstrated to markedly reduce MPTP-induced DA neuronal degeneration $(73,74)$. One study found that selective and non-selective COX-2 inhibitors reduce the rigidity of PD rats, however, the rigidity recovery following administration of the selective COX-2 inhibitor was greater than that of the rats in the non-selective COX-2 inhibitor group (75). Additionally, COX-2 deletion has been shown to moderately attenuate the motor impairment and alteration of dopamine utilization induced by paraquat in the striatum of mice (76). The KO of COX-2 and neuronal NOS in MPTP-induced PD mice reduced the number of positive neurons, indicating degenerative DA, compared with the number observed in wild-type littermates (77). Although the mechanism of the COX-2-mediated DA neuronal degeneration remains unclear, it is hypothesized that COX-2 produces PGE2 and reactive oxygen species (ROS), which cause toxic effects via the c-Jun N-terminal kinase (JNK) signaling pathway and PGE2, rather than PGE2 and ROS (73).

Matrix metalloproteinases (MMPs) and PD. MMPs belong to a family of extracellular soluble or membrane-bound endopeptidases, which are involved in reconstituting the extracellular matrix in addition to neuroinflammatory responses, and are predominantly synthesized by microglia, astrocytes and neurons. The activation of MMPs, particularly MMP-3 and MMP-9, may be associated with the pathogenesis of PD.

MMP-3 expression and activation was found to be significantly increased 24 and $48 \mathrm{~h}$ following LPS injection in an LPS-induced model of PD (78). MMP-3 triggers the microglia to produce proinflammatory and cytotoxic molecules in addition to MMP-3, which subsequently contribute to neuronal damage. MMP-3 is increased in various experimental 
models of PD that are induced using selective toxins and inflammation, and inhibition of MMP-3 may attenuate neuronal death (79). MMP-3 activation ultimately leads to the demise of DA neuronal cells (80). In vitro, MMP-3 activates microglia and the $\mathrm{NF}-\kappa \mathrm{B}$ signaling pathway, and increase the expression levels of TNF- $\alpha$, subsequently causing neuronal apoptosis (81). MMP-3 digests $\alpha$-synuclein in DA neurons, which is critical in the progression of PD via the modulation of $\alpha$-synuclein in aggregation, Lewy body formation and neurotoxicity (82). MMP-3 promotes neuronal degeneration via the release of TNF- $\alpha$ and the activation of the NF- $\kappa \mathrm{B}$ signaling pathway. KO of MMP-3 markedly reduced nigrostriatal DA neuronal degeneration, microglial activation and superoxide generation in an MPTP-induced mouse model of PD (83). Thus, the intracellular active form of MMP-3 is directly associated with apoptotic signaling in DA cells (84).

A study of post-mortem PD brains reported that various MMPs were upregulated in the substantia nigra (80). Lorenzl et al (85) found that the activation of MMP-9 was significantly increased in the striatum and substantia nigra of an MPTP-induced mouse model of PD compared with that observed in the control. Furthermore, the inhibition of MMP-9 significantly attenuated the dopamine depletion and loss of $\mathrm{TH}$ immunoreactive neurons in the SNPC (86). MMP-1 and MMP-3, although not MMP-9, increased the tendency of $\alpha$-synuclein to aggregate, which indicated that MMP-1 and MMP-3 may influence the pathogenesis of PD in vivo by generating specific aggregation-enhancing $\alpha$-synuclein fragments resulting from limited proteolysis.

All results indicate that MMP-3 and MMP-9 are critical in the progression of $\mathrm{PD}$, and that the suppression of MMP-3 and MMP-9 may provide a novel therapeutic strategy for PD.

Toll-like receptors (TLRS). TLRs are key mediators of innate immunity, and their activation leads to the production of cytokines and INFs. There are 10-15 types of TLRs in the majority of mammalian species. Nomura et al (87) first described human TLRs in 1994. In humans, 13 TLRs have been identified, TLR1 to TLR13 (88).

TLRs have been shown to recognize a number of pathogen-associated molecules, including the bacterial cell wall components peptidoglycan (TLR2), dsRNA (TLR3) and ssRNA (TLR7), in addition to non-methylated cytosine-guanosine DNA (TLR9). Additionally, they have been shown to initiate innate immune responses upon interaction with infectious agents. TLRs are situated in antigen-presenting cells, such as dendrites, monocytes/macrophages and microglia in the CNS, and they are considered to be key molecules, which alert the immune system to the presence of microbial infections. Through regulation of the expression and activities of signal-dependent transcription factors, including NF- $\kappa \mathrm{B}$, activator protein-1 and the interferon regulating factor family, TLRs induce inflammatory gene expression. Further experiments have revealed that TLRs are important in the development of numerous diseases in which inflammation has a pathogenic role (89).

TLR3 is expressed throughout the CNS on cerebral endothelial cells, microglia, oligodendrocytes, neurons and most prominently on astrocytes (90). Lehnardt et al (91) demonstrated that TLR4 is critical in LPS-induced neuronal injury in vivo, and indicated that neurons experiencing a single reversible insult undergo irreversible injury following a second insult (microglial activation) and that the specific activation of innate immunity, which is induced by a TLR4-dependent signaling pathway may cause neurodegeneration in the CNS. Panaro et al (92) indicated that compared with untreated animals, augmented expression levels of TLR4 were observed in the substantia nigra of MPTP-treated mice. Noelker et al (93) demonstrated that TLR4-deficient mice are less vulnerable to MPTP intoxication than wild-type mice, in addition to displaying a reduced number of ionized calcium-binding adaptor molecule 1(+) and major histocompatibility complex II(+) activated microglial cells following MPTP application. Kim et al (94) demonstrated that extracellular $\alpha$-synuclein released from neuronal cells is an endogenous agonist for TLR2, which activates inflammatory responses in microglia. Furthermore, it has been demonstrated that TLR2 is upregulated in animal models of PD (95).

Numerous studies have demonstrated that TLR signaling pathways may be novel therapeutic targets for modification of neuroinflammation in PD and associated neurological diseases. However, it is yet undetermined whether certain TLRs found in other mammals are also present in humans. For example, TLR11, -12 and -13 are not observed in humans, however, they are all expressed in mice. Additionally, other TLRs, which are not found in mammals may be found in humans. Therefore, using experimental animals as models of human innate immunity may be complex

NADPH oxidase. The NADPH oxidase is a multicomponent enzyme composed of the following: At least three cytosolic subunits, $\mathrm{p} 47^{\text {Phox }}, \mathrm{p} 67^{\text {Phox }}$ and $\mathrm{p} 40^{\text {Phox }}$; two membrane associated subunits, p22 ${ }^{\text {Phox }}$ and gp91 ${ }^{\text {Phox }}$; and Rac, a small GTPase (96). NADPH oxidase is inactive in resting microglia as $\mathrm{p} 47^{\text {Phox }}$, $\mathrm{p} 67^{\text {Phox }}$ and $\mathrm{p} 40^{\text {Phox }}$, which are present in the cytosol, are segregated from the two transmembrane bound subunits, gp91 ${ }^{\text {Phox }}$ and $\mathrm{p} 22^{\text {Phox }}$. In the presence of microglial activation, $\mathrm{p} 47^{\text {Phox }}$ is phosphorylated and the entire cytosolic complex translocates to the membrane, where electrons are transferred from NADPH to oxygen, which assembles a cytosolic complex with gp91 ${ }^{\text {Phox }}$ and p $22^{\text {Phox }}$, thus forming a complete NADPH oxidase entity that is able to reduce oxygen to a superoxide radical $\left(\mathrm{O}_{2}^{-}\right)$, which subsequently facilitates the production of other secondary reactive oxidants (97). The mesencephalic DA neuronal cells (N27) express key NADPH oxidase subunits, gp91 ${ }^{\text {Phox }}$ and p6 $7^{\text {Phox }}$, hence co-treatment with 4-(2-aminoethyl)benzenesulfonyl fluoride hydrochloride, apocynin or diphenylene iodinium (three structurally diverse NADPH oxidase inhibitors) suppress 1-methyl-4-phenylpyridinium (MPP)(+)-induced cytotoxic cell death and reduce the MPP(+)-mediated increase in caspase-3 enzymatic activity (98). The expression levels of NADPH oxidase 1 increase in a concentration-dependent manner in Paraquat-treated rat N27 DA cells, and pretreatment with apocynin, a putative NADPH oxidase inhibitor, markedly reduces DA cell death (99). In an MPTP-induced PD mouse model, the enhanced gp91 ${ }^{\text {Phox }}$ immunoreactivity was observed specifically in the SNPC and striatum, and gp91 ${ }^{\mathrm{PHOX}}$ protein levels clearly increased in post-mortem SNPC samples from PD patients (100). In gp91 ${ }^{\text {Phox }}$ subtype mutant mice treated with MPTP, the density of striatal TH-positive nerve fibers was less reduced when compared with that in the wild-type littermates (100). 
Heat shock proteins (HSPS) and their anti-inflammatory functions. HSPs are soluble intracellular proteins that mediate a range of essential housekeeping and cytoprotective functions. Their expression levels are increased when cells are exposed to high temperatures or various other types of stress. The endogenous HSPs may have a beneficial effect on PD through the suppression of $\alpha$-synuclein aggregation (101).

Among HSPs, HSP90 and HSP70 have particularly important cytoprotective roles in various pathomechanisms, including the progression of PD. Previous studies have revealed that as a putative anti-apoptotic factor, HSP70 protects against neuronal cell death in PD $(102,103)$ and the application of HSP70 in SK-N-SH cells reduces neurotoxicity by suppressing the elevation of MPP(+)-induced $\alpha$-synuclein levels; furthermore, application of HSP70 prevents DA neuronal loss in $\alpha$-synuclein transgenic Drosophila (104). A study by Tantucci et al (100) investigated a rotenone-induced model of PD, obtaining corticostriatal slices from rats that had undergone mild thermal stress and using them to demonstrate that increased HSP70 reduces rotenone-induced neurotoxicity (105). Additionally, HSP70 inhibits rotenone-mediated apoptosis in the SH-SY5Y human DA cell line in vitro (106), and attenuates nigral injury induced by MPTP in vivo by suppressing proapoptotic factors and activating the survival pathway (107). HSP70 strongly inhibits $\alpha$-synuclein fibril formation via preferential binding of prefibrillar species. Furthermore, HSP70 alters the characteristics of toxic $\alpha$-synuclein aggregates indicating that cellular toxicity arises from the prefibrillar forms of $\alpha$-synuclein (108). In a cellular model of PD, suppression of DJ-1-induced $\alpha$-synuclein toxicity was associated with an increase in the levels of the stress-induced form of HSP70. Additionally, research on RNA interference also revealed that an increase of HSP70 levels was necessary for DJ-1-mediated inhibition of $\alpha$-synuclein aggregation, but not toxicity (109). These results indicate that the induction of HSP70 may be a neuroprotective mechanism in neurodegenerative disorders.

HSP90 resolves disordered protein aggregates and cross- $\beta$ amyloid conformers, and is associated with elevated levels of insoluble $\alpha$-synuclein in PD brains (110). These variations of HSP90 were identified by the neuropathological findings in an $\alpha$-synuclein mutant transgenic PD mouse model (111). The levels of phosphorylated HSP $90 \alpha / \beta$ are significantly increased in the substantia nigra of subjects with PD compared with normal control subjects (112). Wang et al (113) demonstrated that HSP90 in vivo forms a complex with leucine-rich repeat kinase 2 (LRRK2), the mutation of which usually leads to PD, and the association may be disrupted by HSP90 inhibitors, subsequently causing proteasomal degradation of LRRK2. Inhibition of HSP90 prevents resecretion of extracellular $\alpha$-synuclein, which is important in the pathogenesis of PD, and attenuates extracellular $\alpha$-synuclein-induced neurotoxicity (114).

In conclusion, these studies have demonstrated that HSPs may be a promising target for the treatment of PD.

\section{Transcriptional regulation of inflammatory processes}

Transcription factors in $P D$. It is well known that variations in gene expression normally occur in PD. Activation of various transcription factors has been demonstrated in experimental
PD models, particularly with regard to the inflammatory responses in PD.

$N F-\kappa B$ and inflammatory responses. NF- $\kappa \mathrm{B}$ participates in the regulation of inflammatory responses. It is a dimeric transcription factor and one of five proteins in the NF- $\mathrm{KB}$ family, which consists of; Rel (cRel), RelA (p65), RelB, NF-кB1 (p50 and its precursor, p105) and NF- $\mathrm{kB}$. The most frequent form of NF- $\mathrm{KB}$ is a heterodimer consisting of Rel A (p65) and p50. NF- $\mathrm{KB}$ is usually bound to a member of a family of inhibitor proteins

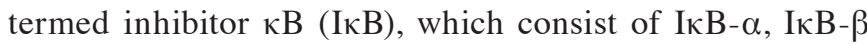

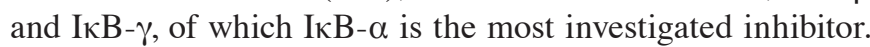
Through an upstream IкB kinase (IKK), the ubiquitination and degradation of I $\mathrm{I} B-\alpha$ is caused by phosphorylation at serines 32 and 36 in the $26 \mathrm{~S}$ proteasome, which frees NF- $\mathrm{KB}$ and enables it to translocate to the nucleus and bind to $\mathrm{\kappa B}$ sites in the initiators of downstream genes to activate their transcription (33).

Numerous functional $\kappa \mathrm{B}$ sites, including intercellular adhesion molecule- 1, TNF- $\alpha$, iNOS and IL- 6 are contained in genes involved in inflammation. In vitro experiments have demonstrated clear upregulation of basal NF- $\mathrm{kB}$ activation in an MPP(+)-induced model of PD in an SH-SY5Y human neuroblastoma cybrid (mitochondrial transgenic cytoplasmic hybrid) cell line (115). By contrast, the impairment of NF- $\mathrm{kB}$ function due to the IKK inhibitor, sulfasalazine, was paralleled by a decline in neurotoxic mediators in the study of human neuromelanin-treated microglia cultures (116). In the MPP(+)-lesioned S-type human neuroblastoma cell line, SH-EP1, NF- $\mathrm{BB}$ was activated by $\mathrm{MPP}(+)$ as evidenced by NF- $\kappa \mathrm{B}$ p65 nuclear translocation, increased DNA binding activity and a rapid phosphorylation of the NF- $\kappa B$ inhibitor, I $\kappa \mathrm{B} \alpha$. NF- $\kappa \mathrm{B}$ partially mediated the neurotoxicity of MPP(+) (117). Recombinant cell-permeable

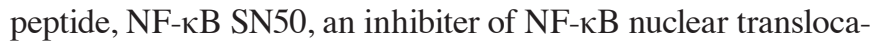
tion, was applied to the dopamine-treated $\mathrm{PC} 12$ cell line and clearly prevented the cells from undergoing apoptosis, which may be associated with PC12 apoptotic induction (118). This finding concurs with the study of Wang et al (119) in which it was observed that complex I deficiency in dopamine cells may induce apoptosis via a process associated with NF- $\mathrm{KB}$ nuclear translocation. Weingarten et al (120) found that a rapid and non-oxidative activation of the stress-inducible transcriptional NF- $\kappa B$ may be associated with mechanisms of NF- $\kappa B$-mediated progressive deterioration of DA neurons in PD (121). In rodent models of PD, NF- $\kappa \mathrm{B}$ was activated in DA neurons $12 \mathrm{~h}$ following 6-OHDA administration and increased immunoreactivities of the NF- $\mathrm{kB}$ target gene, $\mathrm{p} 53$ were observed in the DA neurons. SN50 inhibits NF- $\kappa B$ nuclear translocation and p53 induction (122), in addition, the loss of nigral DA neurons may be reduced by SN50 and the p53 antagonist (122). This indicates that active NF- $\mathrm{KB}$ leads to loss of DA neurons via the NF- $\mathrm{kB}$-dependent $\mathrm{p} 53$-signaling pathway. SN50 was also revealed to alleviate cytotoxicity induced by $\mathrm{MPP}(+)(123)$. Pyrrolidine dithiocarbamate (PDTC), another NF- $\kappa B$ inhibitor, significantly ameliorated the DA deficits induced by MPTP in wild-type mice, where MPTP treatment significantly increased oxidative damage. Consistently, PDTC significantly attenuated MPTP-induced behavioral impairments in wild-type mice (124). Activated NF- $\mathrm{kB}$ expression levels were significantly increased in the striatum of MPTP-treated PD mouse models (125). Aoki et al (29) demonstrated that by activating NF- $\kappa \mathrm{B}$ in 
reactive astrocytes of the substantia nigra, increased cytokines and apoptosis-associated proteins induced apoptosis and caused DA neuronal loss following MPTP treatment in mice. Mogi et al (54) reported that in different nigrostriatal DA regions of patients with $\mathrm{PD}$, including the caudate nucleus, putamen and substantia nigra, expression of $\mathrm{NF}-\kappa \mathrm{B}$ was significantly enhanced. Through the induction of suppressed I $\mathrm{B} \alpha$ expression and the suppression of translocation of the $\mathrm{NF}-\kappa \mathrm{B}$ subunit, p65 to the nucleus in DA neurons, DA cell loss was significantly attenuated in MPTP-mediated PD mouse models (126). In the substantia nigra of PD patients, transcriptional activation of $\mathrm{NF}-\kappa \mathrm{B}$ is considered to be beneficial to the pathomechanism of DA neuronal injury (127). Cao et al (128) found that in early 6-OHDA-induced PD rat models, the NF- $\mathrm{B}$ signaling pathway may be activated by GDNF, and that the nuclear NF- $\kappa$ B p65/p52 complex levels increased following administration of GDNF, while the p65/p50 complex levels reduced. Curcumin was found to inhibit 6-OHDA-induced cell death in MES23.5 cells via modulation of NF- $\kappa$ B translocation (129).

$D J-1$. DJ-1 was initially identified as a novel oncogene and has recently been found to be a causative gene of familial PD, particularly PARK7, mutations of which cause an autosomal recessive, early onset, familial form of $\mathrm{PD}$ (130). It is a multifunction protein, which has a key antioxidant and neuroprotective role in neuronal cells (131-134). In primary dopamine neurons in the N27 rat DA cell line, overexpression of wild-type DJ-1 protected cells from death induced by hydrogen peroxide and 6-OHDA. By contrast, endogenous DJ-1 knock-down renders cells more susceptible to oxidative damage (135).

Deficiency of DJ-1 orthologous genes, DJ-1 $\alpha$ and DJ-1 $\beta$ renders Drosophila vulnerable to paraquat-induced oxidative stress, motor impairment and reduces life span (136). The morphology of DA cell loss differs between DJ-1 KO and wild-type mice (137-139). However, no overt DA neuronal degeneration was observed in aged DJ-1 KO mice $(140,141)$. In DJ-1 KO astrocyte cultures, when treated with TLR4 agonist LPS, the astrocytes generated $>10$ times the amount of NO compared with that of the littermate controls. Proinflammatory mediators, including COX-2 and IL-6, were also significantly induced by DJ-1 KO astrocytes (142). DJ-1 KO in older mice exhibited reduced bodyweight and grip strength, and a more severe gait disorder compared with those of the wild-type littermates (143). Using neuron-astrocyte contact and non-contact co-cultures, Mullett et al $(144,145)$ found that compared with wild-type astrocytes, the neuroprotective capacity against the neurotoxin rotenone was markedly reduced in DJ-1 knock-down asctrocytes. By contrast, DJ-1 overexpression in astrocytes has also been shown to significantly increase their neuroprotective capacity against other neurotoxins. In a cellular model of PD, expression of wild-type DJ-1 was shown to protect primary DA neurons from toxicity caused by rotenone, mutant $\alpha$-synuclein and proteasome inhibitors. Neurons with significantly reduced levels of endogenous DJ-1 are more sensitive to neurotoxins, and DJ-1 mutants involved in familial PD also exhibit a reduced neuroprotective capacity (146).

Upregulation of DJ-1 has been observed in the reactive astrocytes of the human PD brain. Furthermore, in other chronic neurodegenerative diseases and in an $\alpha$-synuclein transgenic mouse model of PD, astroglial DJ-1 was overtly upregulated (147). An upregulation of the expression levels of DJ-1 in the CSF in PD patients was observed when compared with non-PD control subjects (148). By performing quantitative polymerase chain reaction and western blot analysis in numerous post-mortem PD and control brain regions, Kumaran et al (149) found a region-specific reduction in the DJ-1 mRNA expression levels in the putamen, frontal cortex, parietal cortex and cerebellum in PD $(\sim 30-60 \%)$ compared with the levels in the control samples, while a significant upregulation was observed in the amygdaloid nucleus $(\sim 90 \%)$ and entorhinal cortex $(\sim 39 \%)$. The expression levels of total DJ-1 and its reactivity were significantly reduced in the substantia nigra of 18 rapidly autopsied PD brains compared with those observed in nine non-pathological controls, and a reduction in the level of expression of DJ-1 protein was observed in the substantia nigra of sporadic PD patients compared with the levels in control subjects (150). In contrast to the expression of the DJ-1 protein, expression of DJ-1 mRNA was observed in all nigra dopamine neurons, but not in the astrocytes (151). The reason why DJ-1 mRNA is not expressed in astrocytes requires further investigation. The mechanism of action of DJ-1 in PD has not been completely elucidated, however, certain studies $(152,153)$ have found that DJ-1 counteracts dopamine toxicity, and that the overexpression of DJ-1 reduced intracellular ROS. The mitogen activated protein (MAP) kinase signaling pathway mediates the upregulation of DJ-1 via activation of extracellular signal-regulated kinase (ERK) 1 and 2 in vitro and in vivo. Upregulation of DJ-1 is also induced by oxidative stress through the MAP kinase signaling pathway $(154,155)$.

These findings indicate that DJ-1 function disorder leads to an excessive neuroinflammatory response, which may contribute to the pathogenesis of PD. Additionally, the widespread variations of DJ-1 expression levels also demonstrate that DJ-1 function disorder may be associated with the motor and non-motor symptoms of PD. However, it is yet to be elucidated if these changes are specific to PD.

NR4A. The NR4A subfamily are nuclear receptors, which were initially characterized as growth factor-inducible genes and include Nur77 (NR4A1), Nurr1 (NR4A2) and Nor-1 (NR4A3). These receptors are critical in the differentiation of DA neurons.

Members of the NR4A family have been reported to positively and negatively regulate pro-inflammatory genes $(156,157)$. All three NR4A members are strongly induced by inflammatory stimuli and bind to the same response elements, hence they may regulate the expression of the same genes (158). In response to treatment with 12-O-tetradecanoylphorbol-13-acetate or LPS and the inflammatory cytokines, TNF $\alpha$, IFN $\gamma$ and IL-1 $\beta$, expression of Nor-1 (NR4A3) was observed to be clearly induced in the human monocytic cell line, THP-1. Marginal upregulation of Nor-1 induced by macrophage colony-stimulating factor (CSF) and granulocyte macrophage CSF has also been observed in THP-1 cells (158). A significant increase in the expression levels of Nur77 has been observed in the substantia nigra/ventral tegmental area complex following administration of antagonists of dopamine D2 receptors (159). Gilbert et al (160) determined that Nur77-deficient mice exhibited more spontaneous locomotor activity, greater sensitivity to dopamine D2 receptor agonist and increased dopamine metabolite DOPAC, when compared with that in wild-type mice. 
In addition to Nor-1 and Nur77, Nurr1 suppresses the expression of pro-inflammatory neurotoxic mediators in microglia and astrocytes. Reduction of Nurr1 expression levels causes inflammatory responses in microglia, resulting in the death of TH expressing neurons. Additionally, Nurr1 protects against the loss of DA neurons in PD. In Nurr1 KO mice, Nurrl deficiency impairs DA function and increases the degeneration of midbrain DA neurons in PD (161). In vitro and in vivo, reduction of Nurrl expression increases the susceptibility to DA neuronal injury induced by the impairment of the ubiquitin-proteasome system (162). Following LPS stimulation, the expression levels of Nurrl were markedly increased, which could be blocked by inhibitors of ERK, JNK and PI3K/Akt. The ERK inhibitor was shown to partially block the translocation of Nurrl from the cytoplasm to the nucleus (163). Bensinger and Tontonoz (164) also indicated that Nurrl protects DA neurons by suppressing inflammatory gene expression in astrocytes and microglia.

In conclusion, the above-mentioned studies revealed that these receptors may have numerous roles in the inflammatory responses, which occur during the development of PD.

\section{Conclusion}

In conclusion, inflammatory changes may be detected during the pathological development of PD prior to patients succumbing to the disease. However, whether the inflammatory responses are due to an autoimmune response or a secondary event in PD has yet to be determined. In particular, whether the immune response/inflammation is beneficial or destructive to $\mathrm{PD}$ requires further investigation to elucidate their association with the progression of PD. Future studies should focus on the optimal time at which to intervene for the most effective management of inflammation. Furthermore, increasing experimental data are providing novel therapeutic strategies for preventing the neurodegeneration in patients with $\mathrm{PD}$, which reduces the requirement for multiple unnecessary trials, and improves the quality of life for patients with PD and their families.

\section{Acknowledgements}

The present review was supported by a Grant-In-Aid from The First Affiliated Hospital of Henan University of Science and Technology (Luoyang, China) and National Natural Science Foundations of China (grant no. U1304809) from J.Y.

\section{References}

1. Lang A, E., Lozano A, M: Parkinson's disease. First of two parts. N Engl J Med 339: 1044-1053, 1998

2. Anandhan A, Essa MM and Manivasagam T: Therapeutic attenuation of neuroinflammation and apoptosis by black tea theaflavin in chronic MPTP/probenecid model of Parkinson's disease. Neurotox Res 23: 166-173, 2013.

3. Barnum CJ and Tansey MG: Neuroinflammation and non-motor symptoms: the dark passenger of Parkinson's disease? Curr Neurol Neurosci Rep 12: 350-358, 2012.

4. Sekiyama K, Sugama S, Fujita M, et al: Neuroinflammation in Parkinson's disease and related disorders: A lesson from genetically manipulated mouse models of $\alpha$-synucleinopathies. Parkinsons Dis 2012: 271732, 2012.

5. Hirsch EC, Vyas S and Hunot S: Neuroinflammation in Parkinson's disease. Parkinsonism Relat Disord 18 (Suppl 1): S210-S212, 2012.
6. Khan MM, Kempuraj D, Thangavel R and Zaheer A: Protection of MPTP-induced neuroinflammation and neurodegeneration by Pycnogenol. Neurochem Int 62: 379-388, 2013.

7. Ghosh A, Kanthasamy A, Joseph J, et al: Anti-inflammatory and neuroprotective effects of an orally active apocynin derivative in pre-clinical models of Parkinson's disease. J Neuroinflammation 9: 241, 2012.

8. Yan J, Xu Y, Zhu C, et al: Simvastatin prevents dopaminergic neurodegeneration in experimental parkinsonian models: the association with anti-inflammatory responses. PLoS One 6: e20945, 2011.

9. Ghosh A, Roy A, Matras J, Brahmachari S, Gendelman HE and Pahan K: Simvastatin inhibits the activation of p21ras and prevents the loss of dopaminergic neurons in a mouse model of Parkinson's disease. J Neurosci 29: 13543-13556, 2009.

10. Depboylu C, Stricker S, Ghobril JP, Oertel WH, Priller J and Höglinger GU: Brain-resident microglia predominate over infiltrating myeloid cells in activation, phagocytosis and interaction with T-lymphocytes in the MPTP mouse model of Parkinson disease. Exp Neurol 238: 183-191, 2012.

11. Dutta G, Barber DS, Zhang P, Doperalski NJ and Liu B: Involvement of dopaminergic neuronal cystatin $\mathrm{C}$ in neuronal injury-induced microglial activation and neurotoxicity. J Neurochem 122: 752-763, 2012.

12. Long-Smith CM, Sullivan AM and Nolan YM: The influence of microglia on the pathogenesis of Parkinson's disease. Prog Neurobiol 89: 277-287, 2009.

13. Kim WG, Mohney RP, Wilson B, Jeohn GH, Liu B and Hong JS: Regional difference in susceptibility to lipopolysaccharide-induced neurotoxicity in the rat brain: role of microglia. J Neurosci 20: 6309-6316, 2000.

14. Whitton PS: Inflammation as a causative factor in the aetiology of Parkinson's disease. Br J Pharmacol 150: 963-976, 2007.

15. Crotty S, Fitzgerald P, Tuohy E, et al: Neuroprotective effects of novel phosphatidylglycerol-based phospholipids in the 6-hydroxydopamine model of Parkinson's disease. Eur J Neurosci 27: 294-300, 2008.

16. Mirza B, Hadberg H, Thomsen P and Moos T: The absence of reactive astrocytosis is indicative of a unique inflammatory process in Parkinson's disease. Neuroscience 95: 425-432, 2000.

17. Hunot S, Vila M, Teismann P, et al: JNK-mediated induction of cyclooxygenase 2 is required for neurodegeneration in a mouse model of Parkinson's disease. Proc Natl Acad Sci USA 101: 665-670, 2004.

18. Arimoto T and Bing G: Up-regulation of inducible nitric oxide synthase in the substantia nigra by lipopolysaccharide causes microglial activation and neurodegeneration. Neurobiol Dis 12: 35-45, 2003.

19. Bartosz G: Peroxynitrite: mediator of the toxic action of nitric oxide. Acta Biochim Pol 43: 645-659, 1996.

20. Orr CF, Rowe DB and Halliday GM: An inflammatory review of Parkinson's disease. Prog Neurobiol 68: 325-340, 2002.

21. Iravani MM, Leung CC, Sadeghian M, Haddon CO, Rose S and Jenner P: The acute and the long-term effects of nigral lipopolysaccharide administration on dopaminergic dysfunction and glial cell activation. Eur J Neurosci 22: 317-330, 2005.

22. McLaughlin P, Zhou Y, Ma T, et al: Proteomic analysis of microglial contribution to mouse strain-dependent dopaminergic neurotoxicity. Glia 53: 567-582, 2006.

23. Wersinger $C$ and Sidhu A: An inflammatory pathomechanism for Parkinson's disease? Curr Med Chem 13: 591-602, 2006.

24. Stoll G, Jander S and Schroeter M: Cytokines in CNS disorders: neurotoxicity versus neuroprotection. J Neural Transm Suppl 59: 81-89, 2000.

25. Wang DD and Bordey A: The astrocyte odyssey. Prog Neurobiol 86: 342-367, 2008.

26. Vila M, Jackson-Lewis V, Guégan C, et al: The role of glial cells in Parkinson's disease. Curr Opin Neurol 14:483-489, 2001

27. Ortinski PI, Dong J, Mungenast A, et al: Selective induction of astrocytic gliosis generates deficits in neuronal inhibition. Nat Neurosci 13: 584-591, 2010.

28. Mythri RB, Venkateshappa C, Harish G, et al: Evaluation of markers of oxidative stress, antioxidant function and astrocytic proliferation in the striatum and frontal cortex of Parkinson's disease brains. Neurochem Res 36: 1452-1463, 2011.

29. Aoki E, Yano R, Yokoyama H, Kato H and Araki T: Role of nuclear transcription factor kappa B (NF-kappaB) for MPTP (1-methyl-4-phenyl-1,2,3,6-tetrahyropyridine)-induced apoptosis in nigral neurons of mice. Exp Mol Pathol 86: 57-64, 2009. 
30. Saura J, Parés M, Bové J, et al: Intranigral infusion of interleukin-1beta activates astrocytes and protects from subsequent 6-hydroxydopamine neurotoxicity. J Neurochem 85 : 651-661, 2003.

31. Bélanger M and Magistretti PJ: The role of astroglia in neuroprotection. Dialogues Clin Neurosci 11: 281-295, 2009.

32. Sortwell CE, Daley BF, Pitzer MR, McGuire SO, Sladek JR Jr and Collier TJ: Oligodendrocyte-type 2 astrocyte-derived trophic factors increase survival of developing dopamine neurons through the inhibition of apoptotic cell death. J Comp Neurol 426: 143-153, 2000.

33. Dong $\mathrm{Y}$ and Benveniste EN: Immune function of astrocytes. Glia 36: 180-190, 2001

34. Wang Q, Tang XN and Yenari MA: The inflammatory response in stroke. J Neuroimmunol 184: 53-68, 2007.

35. Gomide V, Bibancos T and Chadi G: Dopamine cell morphology and glial cell hypertrophy and process branching in the nigrostriatal system after striatal 6-OHDA analyzed by specific sterological tools. Int J Neurosci 115: 557-582, 2005

36. Wakabayashi K, Hayashi S, Yoshimoto M, Kudo $\mathrm{H}$ and Takahashi H: NACP/alpha-synuclein-positive filamentous inclusions in astrocytes and oligodendrocytes of Parkinson's disease brains. Acta Neuropathol 99: 14-20, 2000.

37. Benarroch EE: Oligodendrocytes: Susceptibility to injury and involvement in neurologic disease. Neurology 72: 1779-1785,2009.

38. Khan O, Filippi M, Freedman MS, et al: Chronic cerebrospinal venous insufficiency and multiple sclerosis. Ann Neurol 67: 286-290, 2010

39. Takagi S, Hayakawa N, Kimoto H, Kato H and Araki T: Damage to oligodendrocytes in the striatum after MPTP neurotoxicity in mice. J Neural Transm 114: 1553-1557, 2007.

40. Rosin C, Colombo S, Calver AA, Bates TE and Skaper SD: Dopamine D2 and D3 receptor agonists limit oligodendrocyte injury caused by glutamate oxidative stress and oxygen/glucose deprivation. Glia 52: 336-343, 2005.

41. McTigue DM and Tripathi RB: The life, death, and replacement of oligodendrocytes in the adult CNS. J Neurochem 107 $1-19,2008$.

42. Kurkowska-Jastrzebska I, Bałkowiec-Iskra E, Ciesielska A, et al: Decreased inflammation and augmented expression of trophic factors correlate with MOG-induced neuroprotection of the injured nigrostriatal system in the murine MPTP model of Parkinson's disease. Int Immunopharmacol 9 : 781-791, 2009

43. Zhao C, Ling Z, Newman MB, Bhatia A and Carvey PM: TNF-alpha knockout and minocycline treatment attenuates blood-brain barrier leakage in MPTP-treated mice. Neurobio Dis 26: 36-46, 2007.

44. Pieper HC, Evert BO, Kaut O, Riederer PF, Waha A and Wüllner U: Different methylation of the TNF-alpha promoter in cortex and substantia nigra: Implications for selective neuronal vulnerability. Neurobiol Dis 32: 521-527, 2008.

45. Rousselet E, Callebert J, Parain K, et al: Role of TNF-alpha receptors in mice intoxicated with the parkinsonian toxin MPTP. Exp Neurol 177: 183-192, 2002.

46. Boka G, Anglade P, Wallach D, Javoy-Agid, et al Immunocytochemical analysis of tumor necrosis factor and its receptors in Parkinson's disease. Neurosci Lett 172: 151-154, 1994.

47. Sriram K, Matheson JM, Benkovic SA, Miller DB, Luster MI and O'Callaghan JP: Deficiency of TNF receptors suppresses microglial activation and alters the susceptibility of brain regions to MPTP-induced neurotoxicity: role of TNF-alpha. FASEB J 20: 670-682, 2006.

48. Sriram K, Matheson JM, Benkovic SA, Miller DB, Luster MI and $\mathrm{O}^{\prime}$ Callaghan JP: Mice deficient in TNF receptors are protected against dopaminergic neurotoxicity: implications for Parkinson's disease. FASEB J 16: 1474-1476, 2002.

49. McCoy MK, Martinez TN, Ruhn KA, et al: Blocking soluble tumor necrosis factor signaling with dominant-negative tumor necrosis factor inhibitor attenuates loss of dopaminergic neurons in models of Parkinson's disease. J Neurosci 26: 9365-9375, 2006.

50. Scalzo P, Kümmer A, Cardoso F and Teixeira AL: Serum levels of interleukin- 6 are elevated in patients with Parkinson's disease and correlate with physical performance. Neurosci Lett 468: 56-58, 2010.

51. Wu DC, Jackson-Lewis V, Vila M, et al: Blockade of microglial activation is neuroprotective in the 1-methyl-4-phenyl-1,2,3,6-tetrahydropyridine mouse model of Parkinson disease. J Neurosci 22: $1763-1771,2002$
52. Ferrari CC, Pott Godoy MC, Tarelli R, Chertoff M, Depino AM and Pitossi FJ: Progressive neurodegeneration and motor disabilities induced by chronic expression of IL-1beta in the substantia nigra. Neurobiol Dis 24: 183-193, 2006.

53. Machado A, Herrera AJ, Venero JL, et al: inflammatory animal model for Parkinson's disease: the intranigral injection of lps induced the inflammatory process along with the selective degeneration of nigrostriatal dopaminergic neurons. ISRN Neurol 2011: 476158, 2011

54. Sawada M, Imamura K and Nagatsu T: Role of cytokines in inflammatory process in Parkinson's disease. J Neural Transm Suppl 70: 373-381, 2006.

55. Nagatsu T and Sawada M: Inflammatory process in Parkinson's disease: role for cytokines. Curr Pharm Des 11: 999-1016, 2005.

56. Mount MP, Lira A, Grimes D, et al: Involvement of interferon-gamma in microglial-mediated loss of dopaminergic neurons. J Neurosci 27: 3328-3337, 2007.

57. Litteljohn D, Mangano E, Shukla N and Hayley S: Interferon-gamma deficiency modifies the motor and co-morbid behavioral pathology and neurochemical changes provoked by the pesticide paraquat. Neuroscience 164: 1894-1906, 2009.

58. Mogi M, Kondo T, Mizuno Y and Nagatsu T: p53 protein, interferon-gamma, and NF-kappaB levels are elevated in the parkinsonian brain. Neurosci Lett 414: 94-97, 2007.

59. Chen H, O'Reilly EJ, Schwarzschild MA and Ascherio A: Peripheral inflammatory biomarkers and risk of Parkinson's disease. Am J Epidemiol 167: 90-95, 2008.

60. Gonzalez-Aparicio R, Flores JA and E. Fernandez-Espejo E: Antiparkinsonian trophic action of glial cell line-derived neurotrophic factor and transforming growth factor betal is enhanced after co-infusion in rats. Exp Neurol 226: 136-147, 2010.

61. Rota E, Bellone G, Rocca P, Bergamasco B, Emanuelli G and Ferrero P: Increased intrathecal TGF-beta1, but not IL-12, IFN-gamma and IL-10 levels in Alzheimer's disease patients. Neurol Sci 27: 33-39, 2006.

62. Rentzos M, Nikolaou C, Andreadou E, et al: Circulating interleukin-10 and interleukin-12 in Parkinson's disease. Acta Neurol Scand 119: 332-337, 2009.

63. Arimoto T, Choi DY, Lu X, et al: Interleukin-10 protects against inflammation-mediated degeneration of dopaminergic neurons in substantia nigra. Neurobiol Aging 28: 894-906, 2007.

64. Qian L, Block ML, Wei SJ, et al: Interleukin-10 protects lipopolysaccharide-induced neurotoxicity in primary midbrain cultures by inhibiting the function of NADPH oxidase. J Pharmacol Exp Ther 319: 44-52, 2006.

65. Sánchez-Capelo A, Colin P, Guibert B, Biguet NF and Mallet $\mathrm{J}$ : Transforming growth factor beta 1 overexpression in the nigrostriatal system increases the dopaminergic deficit of MPTP mice. Mol Cell Neurosci 23: 614-625, 2003.

66. Qian L, Wei SJ, Zhang D, et al: Potent anti-inflammatory and neuroprotective effects of TGF-beta 1 are mediated through the inhibition of ERK and p47 $7^{\text {PHOX }_{-}}-\mathrm{Ser}^{345}$ phosphorylation and translocation in microglia. J Immunol 181: 660-668, 2008.

67. Ross OA, O'Neill C, Rea IM, et al: Functional promoter region polymorphism of the proinflammatory chemokine IL-8 gene associates with Parkinson's disease in the Irish. Hum Immunol 65: 340-346, 2004.

68. Nishimura M, Kuno S, Mizuta I, et al: Influence of monocyte chemoattractant protein 1 gene polymorphism on age at onset of sporadic Parkinson's disease. Mov Disord 18: 953-955, 2003.

69. Shimoji M, Pagan F, Healton EB and Mocchetti I: CXCR4 and CXCL12 expression is increased in the nigro-striatal system of Parkinson's disease. Neurotox Res 16: 318-328, 2009.

70. Edman LC, Mira H, Erices A, et al: Alpha-chemokines regulate proliferation, neurogenesis, and dopaminergic differentiation of ventral midbrain precursors and neurospheres. Stem Cells 26: 1891-1900, 2008.

71. Tsai SJ, Chao CY and Yin MC: Preventive and therapeutic effects of caffeic acid against inflammatory injury in striatum of MPTP-treated mice. Eur J Pharmacol 670: 441-447, 2011.

72. Wang Q, Zheng H, Zhang ZF and Zhang YX: Ginsenoside Rg1 modulates COX-2 expression in the substantia nigra of mice with MPTP-induced Parkinson disease through the P38 signaling pathway. Nan Fang Yi Ke Da Xue Xue Bao 28: 1594-1598, 2008 (In Chinese).

73. Wang Y, Zhang Y, Wei Z, Li H, Zhou H, Zhang Z and Zhang Z: JNK inhibitor protects dopaminergic neurons by reducing COX-2 expression in the MPTP mouse model of subacute Parkinson's disease. J Neurol Sci 285: 172-177, 2009. 
74. Gupta A, Dhir A, Kumar A and Kulkarni SK: Protective effect of cyclooxygenase (COX)-inhibitors against drug-induced catatonia and MPTP-induced striatal lesions in rats. Pharmacol Biochem Behav 94: 219-226, 2009

75. Moghaddam HF, Hemmati A, Nazari Z, Mehrab H, Abid KM and Ardestani MS: Effects of aspirin and celecoxib on rigidity in a rat model of Parkinson's disease. Pak J Biol Sci 10: 3853-3858, 2007.

76. Litteljohn D, Mangano EN and Hayley S: Cyclooxygenase-2 deficiency modifies the neurochemical effects, motor impairment and co-morbid anxiety provoked by paraquat administration in mice. Eur J Neurosci 28: 707-716, 2008.

77. Hoang T, Choi DK, Nagai M, et al: Neuronal NOS and cyclooxygenase-2 contribute to DNA damage in a mouse model of Parkinson disease. Free Radic Biol Med 47: 1049-1056, 2009.

78. McClain JA, Phillips LL and Fillmore HL: Increased MMP-3 and CTGF expression during lipopolysaccharide-induced dopaminergic neurodegeneration. Neurosci Lett 460: 27-31, 2009.

79. Kim EM and Hwang O: Role of matrix metalloproteinase-3 in neurodegeneration. J Neurochem 116: 22-32, 2011

80. Shin EJ, Kim EM, Lee JA, Rhim H and Hwang O: Matrix metalloproteinase-3 is activated by HtrA2/Omi in dopaminergic cells: relevance to Parkinson's disease. Neurochem Int 60: 249-256, 2012.

81. Kim YS, Kim SS, Cho JJ, et al: Matrix metalloproteinase-3: a novel signaling proteinase from apoptotic neuronal cells that activates microglia. J Neurosci 25: 3701-3711, 2005.

82. Choi DH, Kim YJ, Kim YG, Joh TH, Beal MF and Kim YS: Role of matrix metalloproteinase 3-mediated alpha-synuclein cleavage in dopaminergic cell death. J Biol Chem 286: 14168-14177, 2011.

83. Kim YS, Choi DH, Block ML, et al: A pivotal role of matrix metalloproteinase-3 activity in dopaminergic neuronal degeneration via microglial activation. FASEB J 21: 179-187, 2007.

84. Choi DH, Kim EM, Son HJ, et al: A novel intracellular role of matrix metalloproteinase-3 during apoptosis of dopaminergic cells. J Neurochem 106: 405-415, 2008

85. Lorenzl S, Albers DS, Narr S, Chirichigno J and Beal MF: Expression of MMP-2, MMP-9, and MMP-1 and their endogenous counterregulators TIMP-1 and TIMP-2 in postmortem brain tissue of Parkinson's disease. Exp Neurol 178: 13-20, 2002

86. Lorenzl S, Calingasan N, Yang L, et al: Matrix metalloproteinase-9iselevatedin 1-methyl-4-phenyl-1,2,3,6-tetrahydropyridine-induced parkinsonism in mice. Neuromolecular Med 5: 119-132, 2004

87. Nomura N, Miyajima N, Sazuka T, et al: Prediction of the coding sequences of unidentified human genes. I. The coding sequences of 40 new genes (KIAA0001-KIAA0040) deduced by analysis of randomly sampled cDNA clones from human immature myeloid cell line KG-1 (supplement). DNA Res 1: 47-56, 1994.

88. Tabeta K, Georgel P, Janssen E, et al: Toll-like receptors 9 and 3 as essential components of innate immune defense against mouse cytomegalovirus infection. Proc Natl Acad Sci USA 101: 3516-3521, 2004

89. Atkinson TJ: Toll-like receptors, transduction-effector pathways, and disease diversity: evidence of an immunobiological paradigm explaining all human illness? Int Rev Immunol 27: 255-281, 2008.

90. Bsibsi M, Ravid R, Gveric D and van Noort JM: Broad expression of Toll-like receptors in the human central nervous system. J Neuropathol Exp Neurol 61: 1013-1021, 2002.

91. Lehnardt S, Massillon L, Follett P, et al: Activation of innate immunity in the CNS triggers neurodegeneration through a Toll-like receptor 4-dependent pathway. Proc Natl Acad Sci USA 100: 8514-8519, 2003

92. Panaro MA, Lofrumento DD, Saponaro C, et al: Expression of TLR4 and CD14 in the central nervous system (CNS) in a MPTP mouse model of Parkinson's-like disease. Immunopharmacol Immunotoxicol 30: 729-740, 2008

93. Noelker C, Morel L, Lescot T, et al: Toll like receptor 4 mediates cell death in a mouse MPTP model of Parkinson disease. Sci Rep 3 : $1393,2013$.

94. Kim C, Ho DH, Suk JE, et al: Neuron-released oligomeric $\alpha$-synuclein is an endogenous agonist of TLR2 for paracrine activation of microglia. Nat Commun 4: 1562, 2013

95. Letiembre M, Liu Y, Walter S, et al: Screening of innate immune receptors in neurodegenerative diseases: a similar pattern. Neurobiol Aging 30: 759-768, 2009.

96. Groemping Y and Rittinger K: Activation and assembly of the NADPH oxidase: a structural perspective. Biochem J 386: 401-416, 2005.
97. Babior BM: NADPH oxidase: an update. Blood 93: 1464-1476, 1999.

98. Anantharam V, Kaul S, Song C, Kanthasamy A and Kanthasamy AG: Pharmacological inhibition of neuronal NADPH oxidase protects against 1-methyl-4-phenylpyridinium (MPP+)-induced oxidative stress and apoptosis in mesencephalic dopaminergic neuronal cells. Neurotoxicology 28: 988-997, 2007.

99. Cristóvão AC, Choi DH, Baltazar G, Beal MF and Kim YS The role of NADPH oxidase 1-derived reactive oxygen species in paraquat-mediated dopaminergic cell death. Antioxid Redox Signal 11: 2105-2118, 2009.

100. Wu DC, Teismann P, Tieu K, et al: NADPH oxidase mediates oxidative stress in the 1-methyl-4-phenyl-1,2,3,6-tetrahydropyridine model of Parkinson's disease. Proc Natl Acad Sci USA 100: 6145-6150, 2003

101. Liangliang X, Yonghui H, Shunmei E, Shoufang G, Wei Z and Jiangying Z: Dominant-positive HSF1 decreases alpha-synuclein level and alpha-synuclein-induced toxicity. Mol Biol Rep 37: $1875-1881,2010$

102. Benn SC and WoolfCJ: Adultneuron survival strategies - slamming on the brakes. Nat Rev Neurosci 5: 686-700, 2004

103. Meriin AB and Sherman MY: Role of molecular chaperones in neurodegenerative disorders. Int J Hyperthermia 21: 403-419, 2005.

104. Auluck PK, Chan HY, Trojanowski JQ, Lee VM and Bonini NM: Chaperone suppression of alpha-synuclein toxicity in a Drosophila model for Parkinson's disease. Science 295: 865-868, 2002.

105. Tantucci M, Mariucci G, Taha E, et al: Induction of heat shock protein 70 reduces the alteration of striatal electrical activity caused by mitochondrial impairment. Neuroscience 163: 735-740, 2009.

106. Pan T, Li X, Xie W, Jankovic J and Le W: Valproic acid-mediated Hsp70 induction and anti-apoptotic neuroprotection in SH-SY5Y cells. FEBS Lett 579: 6716-6720, 2005.

107. Shen HY, He JC, Wang Y, Huang QY and Chen JF: Geldanamycin induces heat shock protein 70 and protects against MPTP-induced dopaminergic neurotoxicity in mice. J Biol Chem 280: 39962-39969, 2005.

108. Dedmon MM, Christodoulou J, Wilson MR and Dobson CM: Heat shock protein 70 inhibits alpha-synuclein fibril formation via preferential binding to prefibrillar species. J Biol Chem 280: 14733-14740, 2005.

109. Liu M, Aneja R, Sun X, et al: Parkin regulates Eg5 expression by Hsp70 ubiquitination-dependent inactivation of c-Jun NH2-terminal kinase. J Biol Chem 283: 35783-35788, 2008.

110. Uryu K, Richter-Landsberg C, Welch W, et al: Convergence of heat shock protein 90 with ubiquitin in filamentous alpha-synuclein inclusions of alpha-synucleinopathies. Am J Pathol 168: 947-961, 2006.

111. Calabrese V, Mancuso C, Ravagna A, et al: In vivo induction of heat shock proteins in the substantia nigra following L-DOPA administration is associated with increased activity of mitochondrial complex I and nitrosative stress in rats: regulation by glutathione redox state. J Neurochem 101: 709-717, 2007.

112. Kulathingal J, Ko LW, Cusack B and Yen SH: Proteomic profiling of phosphoproteins and glycoproteins responsive to wild-type alpha-synuclein accumulation and aggregation. Biochim Biophys Acta 1794: 211-224, 2009

113. Wang L, Xie C, Greggio E, et al: The chaperone activity of heat shock protein 90 is critical for maintaining the stability of leucine-rich repeat kinase 2. J Neurosci 28: 3384-3391, 2008.

114. Liu J,Zhang JP, Shi M, et al: Rab1la and HSP90 regulate recycling of extracellular alpha-synuclein. J Neurosci 29: 1480-1485, 2009.

115. Cassarino DS, Halvorsen EM, Swerdlow RH, et al: Interaction among mitochondria, mitogen-activated protein kinases, and nuclear factor-kappaB in cellular models of Parkinson's disease. J Neurochem 74: 1384-1392, 2000

116. Wilms H, Rosenstiel P, Sievers J, Deuschl G, Zecca L and Lucius R: Activation of microglia by human neuromelanin is NF-kappaB dependent and involves p38 mitogen-activated protein kinase: implications for Parkinson's disease. FASEB J 17: 500-502, 2003

117. Yang HJ, Wang L, Xia YY, Chang PN and Feng ZW: NF-kappaB mediates MPP+ induced apoptotic cell death in neuroblastoma cells SH-EP1 through JNK and c-Jun/AP-1. Neurochem Int 56: 128-134, 2010.

118. Panet H, Barzilai A, Daily D, Melamed E and Offen D: Activation of nuclear transcription factor kappa B (NF-kappaB) is essential for dopamine-induced apoptosis in PC12 cells. J Neurochem 77: 391-398, 2001. 
119. Wang X, Qin ZH, Leng Y, et al: Prostaglandin A1 inhibits rotenone-induced apoptosis in SH-SY5Y cells. J Neurochem 83 1094-1102, 2002.

120. Weingarten P, Bermak J.and Zhou QY: Evidence for non-oxidative dopamine cytotoxicity: potent activation of NF-kappa B and lack of protection by anti-oxidants. J Neurochem 76: 1794-1804, 2001

121. Kenchappa RS and Ravindranath V: Glutaredoxin is essential for maintenance of brain mitochondrial complex I: studies with MPTP. FASEB J 17: 717-719, 2003.

122. Liang ZQ, Li YL, Zhao XL, et al: NF-kappaB contributes to 6-hydroxydopamine-induced apoptosis of nigral dopaminergic neurons through p53. Brain Res 1145: 190-203, 2007.

123. Williams CA, Lin Y, Maynard A and Cheng SY: Involvement of NF kappa B in potentiated effect of mn-containing dithiocarbamates on MPP(+) induced cell death. Cell Mol Neurobiol 33: 815-823, 2013

124. Pranski E, Van Sanford CD, Dalal N, et al: NF- $\kappa B$ activity is inversely correlated to RNF11 expression in Parkinson's disease. Neurosci Lett 547: 16-20, 2013.

125. Dehmer T, Heneka MT, Sastre M, Dichgans J and Schulz JB: Protection by pioglitazone in the MPTP model of Parkinson's disease correlates with I kappa B alpha induction and block of NF kappa B and iNOS activation. J Neurochem 88: 494-501, 2004.

126. Ghosh A, Roy A, Liu X, et al: Selective inhibition of NF-kappaB activation prevents dopaminergic neuronal loss in a mouse model of Parkinson's disease. Proc Natl Acad Sci USA 104: 18754-18759, 2007.

127. Soós J, Engelhardt JI, Siklós L, Havas L and Majtényi K: The expression of PARP, NF-kappa B and parvalbumin is increased in Parkinson disease. Neuroreport 15: 1715-1718, 2004.

128. Cao JP, Wang HJ, Yu JK, Liu HM and Gao DS: The involvement of NF-kappaB p65/p52 in the effects of GDNF on DA neurons in early PD rats. Brain Res Bull 76: 505-511, 2008.

129. Wang J, Du XX, Jiang H and Xie JX: Curcumin attenuates 6-hydroxydopamine-induced cytotoxicity by anti-oxidation and nuclear factor-kappa B modulation in MES23.5 cells. Biochem Pharmacol 78: 178-183, 2009.

130. Bonifati V, Rizzu P, Squitieri F, et al: DJ-1 (PARK7), a novel gene for autosomal recessive, early onset parkinsonism. Neurol Sci 24: $159-160,2003$

131. Taira T, Saito Y, Niki T, Iguchi-Ariga SM, Takahashi K and Ariga $\mathrm{H}$ : DJ-1 has a role in antioxidative stress to prevent cell death. EMBO Rep 5: 213-218, 2004.

132. Inden M, Taira T, Kitamura Y, et al: PARK7 DJ-1 protects against degeneration of nigral dopaminergic neurons in Parkinson's disease rat model. Neurobiol Dis 24: 144-158, 2006.

133. Yanagida T, Takata K, Inden M, et al: Distribution of DJ-1, Parkinson's disease-related protein PARK7, and its alteration in 6-hydroxydopamine-treated hemiparkinsonian rat brain. J Pharmacol Sci 102: 243-247, 2006.

134. Yanagisawa D, Kitamura Y, Inden M, et al: DJ-1 protects against neurodegeneration caused by focal cerebral ischemia and reperfusion in rats. J Cereb Blood Flow Metab 28: 563-578, 2008.

135.Zhou W and Freed CR: DJ-1 up-regulates glutathione synthesis during oxidative stress and inhibits A53T alpha-synuclein toxicity. J Biol Chem 280: 43150-43158, 2005.

136. Lavara-Culebras E and Paricio N: Drosophila DJ-1 mutants are sensitive to oxidative stress and show reduced lifespan and motor deficits. Gene 400: 158-165, 2007.

137. Andres-Mateos E, Perier C, Zhang L, et al: DJ-1 gene deletion reveals that DJ-1 is an atypical peroxiredoxin-like peroxidase. Proc Natl Acad Sci USA 104: 14807-14812, 2007.

138. Goldberg MS, Pisani A, Haburcak M, et al: Nigrostriatal dopaminergic deficits and hypokinesia caused by inactivation of the familial Parkinsonism-linked gene DJ-1. Neuron 45: 489-496, 2005.

139. Kim RH, Smith PD, Aleyasin H, et al: Hypersensitivity of DJ-1-deficient mice to 1-methyl-4-phenyl-1,2,3, 6-tetrahydropyrindine (MPTP) and oxidative stress. Proc Natl Acad Sci USA 102: 5215-5220, 2005.

140. Chen L, Cagniard B, Mathews T, et al: Age-dependent motor deficits and dopaminergic dysfunction in DJ-1 null mice. J Biol Chem 280: 21418-21426, 2005.

141. Yamaguchi $\mathrm{H}$ and Shen J: Absence of dopaminergic neuronal degeneration and oxidative damage in aged DJ-1-deficient mice. Mol Neurodegener 2: 10, 2007.
142. Waak J, Weber SS, Görner K, et al: Oxidizable residues mediating protein stability and cytoprotective interaction of DJ-1 with apoptosis signal-regulating kinase 1 . J Biol Chem 284: 14245-14257, 2009.

143. Chandran JS, Lin X, Zapata A, et al: Progressive behavioral deficits in DJ-1-deficient mice are associated with normal nigrostriatal function. Neurobiol Dis 29: 505-514, 2008.

144. Mullett SJ, Hamilton RL and Hinkle DA: DJ-1 immunoreactivity in human brain astrocytes is dependent on infarct presence and infarct age. Neuropathology 29: 125-131, 2009.

145. Mullett SJ and Hinkle DA: DJ-1 knock-down in astrocytes impairs astrocyte-mediated neuroprotection against rotenone. Neurobiol Dis 33: 28-36, 2009

146. Kang WY, Yang Q, Jiang XF, et al: Salivary DJ-1 could be an indicator of Parkinson's disease progression. Front Aging Neurosci 6: 102, 2014.

147. Shadrach KG, Rayborn ME, Hollyfield JG and Bonilha VL: DJ-1dependent regulation of oxidative stress in the retinal pigment epithelium (RPE). PLoS One 8: e67983, 2013

148.Liu F, Nguyen JL, Hulleman JD, Li L and Rochet JC: Mechanisms of DJ-1 neuroprotection in a cellular model of Parkinson's disease. J Neurochem 105: 2435-2453, 2008.

149. Neumann M, Müller V, Görner K, Kretzschmar HA, Haass C and Kahle PJ: Pathological properties of the Parkinson's disease-associated protein DJ-1 in alpha-synucleinopathies and tauopathies: relevance for multiple system atrophy and Pick's disease. Acta Neuropathol 107: 489-496, 2004.

150. Waragai M, Wei J, Fujita M, et al: Increased level of DJ-1 in the cerebrospinal fluids of sporadic Parkinson's disease. Biochem Biophys Res Commun 345: 967-972, 2006.

151. Kumaran R, Vandrovcova J, Luk C, et al: Differential DJ-1 gene expression in Parkinson's disease. Neurobiol Dis 36: 393-400, 2009

152. Nural H, He P, Beach T, Sue L, Xia W and Shen Y: Dissembled DJ-1 high molecular weight complex in cortex mitochondria from Parkinson's disease patients. Mol Neurodegener 4: 23, 2009.

153.Xie Z, Zhuang X and Chen L: DJ-1 mRNA anatomical localization and cell type identification in the mouse brain. Neurosci Lett 465: 214-219, 2009.

154.Lev N, Roncevic D, Ickowicz D, Melamed E and Offen D: Role of DJ-1 in Parkinson's disease. J Mol Neurosci 29: 215-225, 2006.

155.Lev N, Ickowicz D, Barhum Y, Lev S, Melamed E and Offen D: DJ-1 protects against dopamine toxicity. J Neural Transm 116: 151-160, 2009.

156. Pei L, Castrillo A and Tontonoz P: Regulation of macrophage inflammatory gene expression by the orphan nuclear receptor Nur77. Mol Endocrinol 20: 786-794, 2006.

157. Doi Y, Oki S, Ozawa T, Hohjoh H, Miyake S and Yamamura T: Orphan nuclear receptor NR4A2 expressed in T cells from multiple sclerosis mediates production of inflammatory cytokines. Proc Natl Acad Sci USA 105: 8381-8386, 2008.

158.Pei L, Castrillo A, Chen M, Hoffmann A and Tontonoz P: Induction of NR4A orphan nuclear receptor expression in macrophages in response to inflammatory stimuli. J Biol Chem 280: 29256-29262, 2005.

159. Maheux J, Ethier I, Rouillard C and Lévesque D: Induction patterns of transcription factors of the nur family (nurr1, nur77, and nor-1) by typical and atypical antipsychotics in the mouse brain: implication for their mechanism of action. J Pharmacol Exp Ther 313: 460-473, 2005.

160.Gilbert F, Morissette M, St-Hilaire M, et al: Nur77 gene knockout alters dopamine neuron biochemical activity and dopamine turnover. Biol Psychiatry 60: 538-547, 2006.

161. Jankovic J, Chen S and Le WD: The role of Nurr1 in the development of dopaminergic neurons and Parkinson's disease. Prog Neurobiol 77: 128-138, 2005.

162.Pan T, Zhu W, Zhao H, et al: Nurrl deficiency predisposes to lactacystin-induced dopaminergic neuron injury in vitro and in vivo. Brain Res 1222: 222-229, 2008.

163. Fan X, Luo G, Ming M, et al: Nurr1 expression and its modulation in microglia. Neuroimmunomodulation 16: 162-170, 2009.

164. Bensinger SJ and Tontonoz P: A Nurrl pathway for neuroprotection. Cell 137: 26-28, 2009. 